\title{
InterCriteria Analysis of ACO Start Startegies
}

\author{
Stefka Fidanova \\ IICT BAS \\ Sofia, Bulgaria \\ E-mail: stefka@parallel.bas.bg
}

\author{
Olympia Roeva \\ IBBE BAS \\ Sofia, Bulgaria \\ E-mail: olympia@biomed.bas.bg \\ Marcin Paprzycki \\ SRI PAS \\ Warsaw, Poland \\ E-mail: marcin.paprzycki@ibspan.waw.pl
}

\author{
Pawel Gepner \\ Intel Corporation \\ Swindon, UK \\ E-mail: pawel.gepner@intel.com
}

\begin{abstract}
In combinatorial optimization, the goal is to find the optimal object from a finite set. Since such problems are hard to be solved, usually some metaheuristics is applied. One of the most successful techniques for a number of classes of problems is Ant Colony Optimization (ACO). Some start strategies can be applied, to the ACO algorithms, to improve their performance. Here, the InterCriteria Analysis (ICrA) is applied to the ACO algorithm. On the basis of the ICrA, we examine and analyse the ACO performance according to the different start strategies.
\end{abstract}

\section{INTRODUCTION}

$\mathbf{T}$ HE IDEA for the ACO arises fro the way that real ants look for food. The ACO algorithm was proposed, by Marco Dorigo, more than 20 years [6]. Later, several variants and supplements were added, to improve its performance [6]. In [7], various ACO start strategies, which lead to finding better solutions, were proposed.

The InterCriteria Analysis (ICrA) is aiming at going beyond the nature of the criteria involved in a process of evaluation of multiple objects against multiple criteria, and, thus to discover some dependencies between the ICrA criteria themselves [2]. Initially, the ICrA has been applied in temporal, threshold and trends analyses of an economic case-study of EU member states' competitiveness [4]. Further, the ICrA has been used to discover dependencies between parameters in mathematical models and performance criteria for metaheuristics such as GAs and ACO [1], [9]. Here, the ICrA is applied for analysis of an ACO algorithm, with various start strategies. The Multiple Knapsack Problem (MKP) is used as a test problem. The goal is to analyze the dependence between start strategies and algorithm performance, and correlations between strategies.

\section{ACO Algorithm with Start Strategies}

Let us consider the ACO algorithm applied to the solution of a problem represented by a graph. Here, the feasible solutions are represented by path in that graph. The solution process "compares the length" of available paths. In every iteration, an ant starts from a random node and creates a solution. If the last selected node is $u$, the ant selects the next node $(v)$, to be included in the path, by applying probabilistic rule called transition probability.

$$
p_{u v}=\tau_{u v}^{\alpha} \eta_{u v}^{\beta} /\left[\sum_{(u, w) \in E_{S}: w \not \subset X}\left(\tau_{u w}^{\alpha} \eta_{u w}^{\beta}\right)\right],
$$

Here, $\alpha$ and $\beta$ are transition probability parameters, $\tau \in(0,1)$ is a numerical information called pheromone, and $\eta$ is an heuristic information related to the problem. At the beginning, the value of the pheromone across elements is the same. In each iteration, we update the pheromone, on selected elements of the graph, according to the value of the objective function (elements belonging to the better solutions receive more pheromone than others). The random start is very important for good performance of the ACO algorithm, but for some classes of problems (e.g. subset problems), selection of the starting node can be significant. For better managing the search process we include semi-random start of the ants. Here, the nodes are divided into several subsets. An estimation of how good and how bad is to start from some subset is introduced according the number of good and bad solutions that started from this subset. Assume that $D_{j}$ is the estimation of how good it is to start from subset $j$, and $E_{j}$ is the estimation how bad it is to start from subset $j$ [7].

$$
\begin{aligned}
& D_{j}(i)=\phi \cdot D_{j}(i-1)+(1-\phi) \cdot F_{j}(i), \\
& E_{j}(i)=\phi \cdot E_{j}(i-1)+(1-\phi) \cdot G_{j}(i),
\end{aligned}
$$

where $i \geq 1$ is the current process iteration and, for each $j(1 \leq j \leq N)$ :

$$
\begin{gathered}
F_{j}(i)=\left\{\begin{array}{ll}
f_{j, A} / n_{j} & \text { if } n_{j} \neq 0 \\
F_{j}(i-1) & \text { otherwise }
\end{array},\right. \\
G_{j}(i)=\left\{\begin{array}{ll}
g_{j, B} / n_{j} & \text { if } n_{j} \neq 0 \\
G_{j}(i-1) & \text { otherwise }
\end{array},\right.
\end{gathered}
$$

$f_{j, A}$ is the number of the solutions among the best $A \%, g_{j, B}$ is the number of the solutions among the worst $B \%$, where $A+B \leq 100, i \geq 2$ and $\sum_{j=1}^{N} n_{j}=n$, where $n_{j}(1 \leq j \leq N)$ 
is the number of solutions obtained by ants starting from nodes subset $j, n$ is the number of ants. Here, initial values of the weight coefficients are: $D_{j}(1)=1$ and $E_{j}(1)=0$. Parameter $\phi, 0 \leq \phi \leq 1$, shows the weight of the information from the previous iterations and from the last iteration. Several start strategies and combinations between them are proposed. If thresholds for good estimation $D$ and for bad estimation $E$ are fixed, the proposed start strategies are as follows [7]:

1) If $E_{j}(i) / D_{j}(i)>E$ then, for current iteration, the subset $j$ is forbidden. The starting node is randomly chosen from $\{j \mid j$ is not forbidden $\}$;

2) If $E_{j}(i) / D_{j}(i)>E$ then, for current simulation, the subset $j$ is forbidden. The starting node is randomly chosen from $\{j \mid j$ is not forbidden $\}$;

3) If $E_{j}(i) / D_{j}(i)>E$ then, for $K_{1}$ consecutive iterations, the subset $j$ is forbidden. The starting node is randomly chosen from $\{j \mid j$ is not forbidden $\}$;

4) Let $r_{1} \in\left[\frac{1}{2}, 1\right)$ and $r_{2} \in[0,1]$ to be random numbers. If $r_{2}>r_{1}$, a node from subset $\left\{j \mid D_{j}(i)>D\right\}$ is randomly chosen, otherwise a node from the not forbidden subsets is randomly chosen. $r_{1}$ is chosen and fixed at the beginning.

5) Let $r_{1} \in\left[\frac{1}{2}, 1\right)$ and $r_{2} \in[0,1]$ to be random numbers. If $r_{2}>r_{1}$, a node from subset $\left\{j \mid D_{j}(i)>D\right\}$ is randomly chosen, otherwise a node from the not forbidden subsets is randomly chosen. $r_{1}$ is chosen at the beginning and increase with $r_{3}$ every iteration, $r_{3} \in(0,1)$ is a parameter.

Here $K_{1}, K_{1} \in[0$, number of iterations $]$ is a parameter.

We can apply one of start strategies, or combine some of them. Strategies 1, 2, and 3 can be combined with strategies 4 and 5 . When an ant chooses a start node, first applied strategy is one of 1,2 , or 3 , after that, strategy 5 or 6 is used. Thus, together with a completely random start, there are 12 strategies that can be applied (see, also [7]).

\section{Multiple Knapsack Problem}

The start node selection is very important for the subset problems, because only some nodes of the graph of the problem belong to the solution. The Multiple Knapsack Problem (MKP) is a representative of the class of subset problems. It also arises as a sub-problem in a group of more complex problems. Some of important applications that can be formulated as a MKP are cargo loading problems, cutting stock, bin-packing, budget control and financial management. The MKP is also used in a fault tolerance problem [11]. Authors of [5] designed a public cryptography scheme whose security is based on the difficulty of solving the MKP. In [8] two-processor scheduling problems are proposed to be solved as a MKP. Other applications include industrial management, naval, aerospace, computational complexity theory, etc. The MKP can be formulated as follows:

$$
\begin{gathered}
\max \sum_{j=1}^{n} p_{j} x_{j} \\
\text { subject to } \sum_{j=1}^{n} r_{i j} x_{j} \leq c_{i} \quad i=1, \ldots, m \\
x_{j} \in\{0,1\} \quad j=1, \ldots, n
\end{gathered}
$$

$$
x_{j}=\left\{\begin{array}{l}
1 \text { iff the object } j \text { is chosen } \\
0 \text { otherwise }
\end{array}\right.
$$

where $m$ are the resources (the knapsacks), $n$ are the objects, $p_{j}$ is a profit of every object $j, c_{j}$ (knapsack capacity) is the resource budget, $r_{i j}$ is the consumption of resource $i$ by object $j$. The goal is maximizing the sum of profits for a limited budget.

There are $m$ constraints in this problem, so the MKP is also called $m$-dimensional knapsack problem. Let

$$
I=\{1, \ldots, m\}, J=\{1, \ldots, n\},
$$

with $c_{i} \geq 0$ for all $i \in I$. A well-stated MKP assumes that $p_{j}>0, r_{i j} \leq c_{i} \leq \sum_{j=1}^{n} r_{i j}$ for all $i \in I, j \in J$. Note that the $\left[r_{i j}\right]_{m \times n}$ matrix and the $\left[c_{i}\right]_{m}$ vector are both non-negative.

\section{INTERCRITERIA ANALYSIS}

Let us be given an Index Matrix (IM, [3]) whose index sets for rows consist of the names of the criteria and for columns of objects. We will obtain an IM with index sets consisting of names of the criteria both for rows and for columns. Elements of this IM correspond to the degrees of "agreement" and degrees of "disagreement" of the considered criteria. The following two points are assumed [10]. (1) All criteria provide an evaluation for all objects and all these evaluations are available. (2) All the evaluations of a given criteria can be compared among themselves. Further, by $O$, we denote the set of all objects $O_{1}, O_{2}, \ldots, O_{n}$ being evaluated, and by $C(O)$ the set of values assigned by a given criteria $C$ to the objects. Let $x_{i}=C\left(O_{i}\right)$. Then the following set can be defined:

$$
C^{*}(O) \stackrel{\text { def }}{=}\left\{\left\langle x_{i}, x_{j}\right\rangle \mid i \neq j \&\left\langle x_{i}, x_{j}\right\rangle \in C(O) \times C(O)\right\} .
$$

In order to find the degrees of "agreement" of two criteria, the vector of all internal comparisons of each criteria is constructed. This vector fulfills exactly one of the following three relations: $R, \bar{R}$ and $\tilde{R}$. For a fixed criterion $C$, and any ordered pair $\langle x, y\rangle \in C^{*}(O)$ it is required that: $\langle x, y\rangle \in R \Leftrightarrow\langle y, x\rangle \in \bar{R},\langle x, y\rangle \in \tilde{R} \Leftrightarrow\langle x, y\rangle \notin(R \cup \bar{R})$, $R \cup \bar{R} \cup \tilde{R}=C^{*}(O)$

For a criterion $C$, let us define a preference matrix between objects $1,2, \ldots, n$ so that $C_{i j}$ is 1 if $i$ is better than $j,-1$ if $i$ is worse than $j$, and 0 if $i$ and $j$ are equivalent or incomparable over criterion $C$. We determine the degree of "agreement" $\left(\mu_{C, C^{\prime}}\right)$ between the two criteria as the proportion of matching components. This can be done in several ways, e.g. by counting the matches or by taking the complement of the Hamming distance. The degree of "disagreement" $\left(\nu_{C, C^{\prime}}\right)$ is the proportion of components of opposing signs in the two vectors. The difference $\pi_{C, C^{\prime}}=1-\mu_{C, C^{\prime}}-\nu_{C, C^{\prime}}$ is considered as a degree of "uncertainty".

\section{NuMERICAL RESUltS}

We combined the ICrA with the ACO for different start strategies applied to the MKP [7]. Ten test problems from the "OR-Library" with 100 objects and 10 constraints (available from 
http://people.brunel.ac.uk/ mastjjb/jeb/ orlib) were used. The ACO algorithm is applied to various number of nodes in node subsets. The node subsets consist of the same number of nodes, namely 1, 2, 4, 5 and 10 . The average results over the 10 test problems and 30 runs of every problem with every strategy was obtained. The ranking is from 10 to 100 . The ICrA objects $\left(O_{1}, O_{2}, \ldots, O_{20}\right)$ are the different conditions, namely nodes $1,2,4,5$ and 10 , in four cases of $\phi, \phi=\left[\begin{array}{llll}0 & 0.25 & 0.5 & 0.75\end{array}\right]$. The ICrA criteria $\left(C_{1}, C_{2}, \ldots, C_{12}\right)$ are 12 different start strategies for the ACO. The ICrA resulted in two IM ,with the relations between considered 12 criteria. The resulting IMs, for $\mu_{C, C^{\prime}}$ and $\nu_{C, C^{\prime}}$, are shown in Table I and Table II.

TABLE I: Index matrix for $\mu_{C, C^{\prime}}$

\begin{tabular}{l|rrrrrr} 
& $C_{1}$ & $C_{2}$ & $C_{3}$ & $C_{4}$ & $C_{5}$ & $C_{6}$ \\
\hline$C_{1}$ & 1.000 & 0.042 & 0.016 & 0.079 & 1.000 & 1.000 \\
$C_{2}$ & 0.042 & 1.000 & 0.642 & 0.742 & 0.042 & 0.042 \\
$C_{3}$ & 0.016 & 0.642 & 1.000 & 0.670 & 0.016 & 0.016 \\
$C_{4}$ & 0.079 & 0.742 & 0.670 & 1.000 & 0.079 & 0.079 \\
$C_{5}$ & 1.000 & 0.042 & 0.016 & 0.079 & 1.000 & 1.000 \\
$C_{6}$ & 1.000 & 0.042 & 0.016 & 0.079 & 1.000 & 1.000 \\
$C_{7}$ & 0.037 & 0.826 & 0.653 & 0.758 & 0.037 & 0.037 \\
$C_{8}$ & 0.037 & 0.826 & 0.653 & 0.758 & 0.037 & 0.037 \\
$C_{9}$ & 0.026 & 0.637 & 0.889 & 0.705 & 0.026 & 0.026 \\
$C_{10}$ & 0.026 & 0.621 & 0.884 & 0.700 & 0.026 & 0.026 \\
$C_{11}$ & 0.026 & 0.737 & 0.695 & 0.821 & 0.026 & 0.026 \\
$C_{12}$ & 0.026 & 0.737 & 0.695 & 0.821 & 0.026 & 0.026 \\
& & & & & & \\
& $C_{7}$ & $C_{8}$ & $C_{9}$ & $C_{10}$ & $C_{11}$ & $C_{12}$ \\
\hline$C_{1}$ & 0.037 & 0.037 & 0.026 & 0.026 & 0.026 & 0.026 \\
$C_{2}$ & 0.826 & 0.826 & 0.637 & 0.621 & 0.737 & 0.737 \\
$C_{3}$ & 0.653 & 0.653 & 0.889 & 0.884 & 0.695 & 0.695 \\
$C_{4}$ & 0.758 & 0.758 & 0.705 & 0.700 & 0.821 & 0.821 \\
$C_{5}$ & 0.037 & 0.037 & 0.026 & 0.026 & 0.026 & 0.026 \\
$C_{6}$ & 0.037 & 0.037 & 0.026 & 0.026 & 0.026 & 0.026 \\
$C_{7}$ & 1.000 & 1.000 & 0.695 & 0.679 & 0.800 & 0.800 \\
$C_{8}$ & 1.000 & 1.000 & 0.695 & 0.679 & 0.800 & 0.800 \\
$C_{9}$ & 0.695 & 0.695 & 1.000 & 0.984 & 0.753 & 0.753 \\
$C_{10}$ & 0.679 & 0.679 & 0.984 & 1.000 & 0.747 & 0.747 \\
$C_{11}$ & 0.800 & 0.800 & 0.753 & 0.747 & 1.000 & 1.000 \\
$C_{12}$ & 0.800 & 0.800 & 0.753 & 0.747 & 1.000 & 1.000
\end{tabular}

For better understanding of the results, the values of the $\mu_{C, C^{\prime}}, \nu_{C, C^{\prime}}, \pi_{C, C^{\prime}}$ of the criteria pairs, are sorted by the value of the $\mu_{C, C^{\prime}}$. The list is presented in Tables III and IV. Table III shows the criteria pair with high degrees of "agreement" $\left(\mu_{C, C^{\prime}}\right)$ and low value for the degree of "disagreement" $\left(\nu_{C, C^{\prime}}\right)$. Table IV shows the criteria pair with high degree of "uncertainty". Regarding Tables III and IV we observe that relations between criterion $C_{1}$ and criteria $C_{5}$ and $C_{6}$ have the highest value of $\mu_{C, C^{\prime}}\left(\mu_{C, C^{\prime}}=1\right)$, i.e. these criteria are in strong positive consonance. Henceforth, the ACO algorithm performs in a similar way with random start and start strategies 4 and 5. In strategies 4 and 5 there are no forbidden regions (as in the random start). In these cases, only the probability to choose the next element in the solution is different. Other pairs that have the highest value of $\mu_{C, C^{\prime}}\left(\mu_{C, C^{\prime}}=1\right)$ are $C_{7}-C_{8}$ and $C_{11}-C_{12}$. These strategies (Strategies 1-4, 1-5, 3-4 and 3-5) show also very similar performance.
TABLE II: Index matrix for $\nu_{C, C^{\prime}}$

\begin{tabular}{l|rrrrrr} 
& $C_{1}$ & $C_{2}$ & $C_{3}$ & $C_{4}$ & $C_{5}$ & $C_{6}$ \\
\hline$C_{1}$ & 0.000 & 0.000 & 0.000 & 0.000 & 0.000 & 0.000 \\
$C_{2}$ & 0.000 & 0.000 & 0.300 & 0.137 & 0.000 & 0.000 \\
$C_{3}$ & 0.000 & 0.300 & 0.000 & 0.237 & 0.000 & 0.000 \\
$C_{4}$ & 0.000 & 0.137 & 0.237 & 0.000 & 0.000 & 0.000 \\
$C_{5}$ & 0.000 & 0.000 & 0.000 & 0.000 & 0.000 & 0.000 \\
$C_{6}$ & 0.000 & 0.000 & 0.000 & 0.000 & 0.000 & 0.000 \\
$C_{7}$ & 0.000 & 0.095 & 0.295 & 0.137 & 0.000 & 0.000 \\
$C_{8}$ & 0.000 & 0.095 & 0.295 & 0.137 & 0.000 & 0.000 \\
$C_{9}$ & 0.000 & 0.079 & 0.079 & 0.189 & 0.000 & 0.000 \\
$C_{10}$ & 0.000 & 0.084 & 0.084 & 0.195 & 0.000 & 0.000 \\
$C_{11}$ & 0.000 & 0.263 & 0.263 & 0.084 & 0.000 & 0.000 \\
$C_{12}$ & 0.000 & 0.263 & 0.263 & 0.084 & 0.000 & 0.000 \\
& & & & & & \\
& $C_{7}$ & $C_{8}$ & $C_{9}$ & $C_{10}$ & $C_{11}$ & $C_{12}$ \\
\hline & & & & & & \\
$C_{1}$ & 0.000 & 0.000 & 0.000 & 0.000 & 0.000 & 0.000 \\
$C_{2}$ & 0.095 & 0.095 & 0.295 & 0.311 & 0.195 & 0.195 \\
$C_{3}$ & 0.295 & 0.295 & 0.079 & 0.079 & 0.263 & 0.263 \\
$C_{4}$ & 0.137 & 0.137 & 0.189 & 0.195 & 0.084 & 0.084 \\
$C_{5}$ & 0.000 & 0.000 & 0.000 & 0.000 & 0.000 & 0.000 \\
$C_{6}$ & 0.000 & 0.000 & 0.000 & 0.000 & 0.000 & 0.000 \\
$C_{7}$ & 0.000 & 0.000 & 0.242 & 0.258 & 0.137 & 0.137 \\
$C_{8}$ & 0.000 & 0.000 & 0.242 & 0.258 & 0.137 & 0.137 \\
$C_{9}$ & 0.242 & 0.242 & 0.000 & 0.016 & 0.205 & 0.205 \\
$C_{10}$ & 0.258 & 0.258 & 0.016 & 0.000 & 0.211 & 0.211 \\
$C_{11}$ & 0.137 & 0.137 & 0.205 & 0.211 & 0.000 & 0.000 \\
$C_{12}$ & 0.137 & 0.137 & 0.205 & 0.211 & 0.000 & 0.000
\end{tabular}

The criteria pairs still in a consonance, are pairs of criteria $C_{2}, C_{3}, C_{4}$ and $C_{7}, C_{8}, \ldots, C_{12}$. They correspond to Strategies 1, 2 and 3, combined with 4 and 5. In all this strategies there are forbidden regions, therefore the ACO performs in a similar way when we apply "any of them". The criteria pairs with value of $\mu_{C, C^{\prime}}=[0.75-0.25)$ are in dissonance, i.e. there are no dependencies between these criteria (they are independent). The ACO algorithm with random strategies and the ACO algorithm with strategies with forbidden regions perform in a very different way, thus we can not find relations between them.

\section{CONCLUSION}

In this paper, an ICrA is used with the ACO algorithm, to establish the relations and dependencies between the ACO performance and the start strategies. Twelve start strategies are studied. Part of them disallow some regions of the search space for one or more iterations. We can conclude that criteria corresponding to the strategies without forbidden regions are in positive consonance, as well as the criteria corresponding to the strategies with forbidden regions. The criteria corresponding to the strategies with forbidden regions are in dissonance with criteria corresponding to the strategies without forbidden regions.

\section{ACKNOWLEDGMENT}

Work presented here is partially supported by the National Scientific Fund of Bulgaria under grants DFNI-I02/5 and DFNI I02/20, and by the Polish-Bulgarian collaborative grant "Parallel and Distributed Computing Practices". 
TABLE III: Criteria pairs-I

\begin{tabular}{cccc}
\hline Criteria pairs & $\mu_{C, C^{\prime}}$ & $\nu_{C, C^{\prime}}$ & $\pi_{C, C^{\prime}}$ \\
\hline$C_{1}-C_{5}$ & 1.000 & 0.000 & 0.000 \\
$C_{1}-C_{6}$ & 1.000 & 0.000 & 0.000 \\
$C_{5}-C_{6}$ & 1.000 & 0.000 & 0.000 \\
$C_{7}-C_{8}$ & 1.000 & 0.000 & 0.000 \\
$C_{11}-C_{12}$ & 1.000 & 0.000 & 0.000 \\
$C_{9}-C_{10}$ & 0.984 & 0.016 & 0.000 \\
$C_{3}-C_{9}$ & 0.889 & 0.079 & 0.032 \\
$C_{3}-C_{10}$ & 0.889 & 0.079 & 0.032 \\
$C_{2}-C_{7}$ & 0.826 & 0.095 & 0.079 \\
$C_{2}-C_{8}$ & 0.826 & 0.095 & 0.079 \\
$C_{4}-C_{11}$ & 0.821 & 0.084 & 0.095 \\
$C_{4}-C_{12}$ & 0.821 & 0.084 & 0.095 \\
$C_{7}-C_{11}$ & 0.800 & 0.137 & 0.063 \\
$C_{7}-C_{12}$ & 0.800 & 0.137 & 0.063 \\
$C_{8}-C_{11}$ & 0.800 & 0.137 & 0.063 \\
$C_{8}-C_{12}$ & 0.800 & 0.137 & 0.063 \\
$C_{4}-C_{7}$ & 0.758 & 0.137 & 0.105 \\
$C_{4}-C_{8}$ & 0.758 & 0.137 & 0.105 \\
$C_{9}-C_{11}$ & 0.753 & 0.205 & 0.042 \\
$C_{9}-C_{12}$ & 0.753 & 0.205 & 0.042 \\
$C_{10}-C_{11}$ & 0.747 & 0.211 & 0.042 \\
$C_{10}-C_{12}$ & 0.747 & 0.211 & 0.042 \\
$C_{2}-C_{4}$ & 0.742 & 0.137 & 0.121 \\
$C_{2}-C_{11}$ & 0.737 & 0.195 & 0.068 \\
$C_{2}-C_{12}$ & 0.737 & 0.195 & 0.068 \\
$C_{4}-C_{9}$ & 0.705 & 0.189 & 0.105 \\
$C_{4}-C_{10}$ & 0.700 & 0.195 & 0.105 \\
$C_{3}-C_{11}$ & 0.695 & 0.263 & 0.042 \\
$C_{3}-C_{12}$ & 0.695 & 0.263 & 0.042 \\
$C_{7}-C_{9}$ & 0.695 & 0.242 & 0.063 \\
$C_{8}-C_{9}$ & 0.695 & 0.242 & 0.063 \\
$C_{8}-C_{10}$ & 0.679 & 0.258 & 0.063 \\
$C_{7}-C_{10}$ & 0.679 & 0.258 & 0.063 \\
$C_{3}-C_{4}$ & 0.670 & 0.237 & 0.084 \\
$C_{3}-C_{7}$ & 0.653 & 0.295 & 0.053 \\
$C_{3}-C_{8}$ & 0.653 & 0.295 & 0.053 \\
$C_{2}-C_{3}$ & 0.642 & 0.300 & 0.058 \\
$C_{2}$ & 0.637 & 0.295 & 0.068 \\
& & 0.311 & 0.068
\end{tabular}

\section{REFERENCES}

[1] M. Angelova, O. Roeva, T. Pencheva, InterCriteria Analysis of Crossover and Mutation Rates Relations in Simple Genetic Algorithm, Proceedings of the Federated Conference on Computer Science and Information Systems, Vol. 5, 419-424, 2015.

[2] K. Atanassov, D. Mavrov, V. Atanassova, Intercriteria Decision Making: A New Approach for Multicriteria Decision Making, Based on Index Matrices and Intuitionistic Fuzzy Sets. Issues in IFSs and GNs 11, 18 (2014)
TABLE IV: Criteria pairs- II

\begin{tabular}{cccc}
\hline Criteria pairs & $\mu_{C, C^{\prime}}$ & $\nu_{C, C^{\prime}}$ & $\pi_{C, C^{\prime}}$ \\
\hline$C_{1}-C_{4}$ & 0.079 & 0.000 & 0.921 \\
$C_{4}-C_{5}$ & 0.079 & 0.000 & 0.921 \\
$C_{4}-C_{6}$ & 0.079 & 0.000 & 0.921 \\
$C_{1}-C_{2}$ & 0.042 & 0.000 & 0.958 \\
$C_{2}-C_{5}$ & 0.042 & 0.000 & 0.958 \\
$C_{2}-C_{6}$ & 0.042 & 0.000 & 0.958 \\
$C_{1}-C_{7}$ & 0.037 & 0.000 & 0.963 \\
$C_{1}-C_{8}$ & 0.037 & 0.000 & 0.963 \\
$C_{5}-C_{7}$ & 0.037 & 0.000 & 0.963 \\
$C_{5}-C_{8}$ & 0.037 & 0.000 & 0.963 \\
$C_{6}-C_{7}$ & 0.037 & 0.000 & 0.963 \\
$C_{6}-C_{8}$ & 0.037 & 0.000 & 0.963 \\
$C_{1}-C_{9}$ & 0.026 & 0.000 & 0.974 \\
$C_{1}-C_{10}$ & 0.026 & 0.000 & 0.974 \\
$C_{1}-C_{11}$ & 0.026 & 0.000 & 0.974 \\
$C_{1}-C_{12}$ & 0.026 & 0.000 & 0.974 \\
$C_{5}-C_{9}$ & 0.026 & 0.000 & 0.974 \\
$C_{5}-C_{10}$ & 0.026 & 0.000 & 0.974 \\
$C_{5}-C_{11}$ & 0.026 & 0.000 & 0.974 \\
$C_{5}-C_{12}$ & 0.026 & 0.000 & 0.974 \\
$C_{6}-C_{9}$ & 0.026 & 0.000 & 0.974 \\
$C_{6}-C_{10}$ & 0.026 & 0.000 & 0.974 \\
$C_{6}-C_{11}$ & 0.026 & 0.000 & 0.974 \\
$C_{6}-C_{12}$ & 0.026 & 0.000 & 0.974 \\
$C_{1}-C_{3}$ & 0.016 & 0.000 & 0.984 \\
$C_{3}-C_{5}$ & 0.016 & 0.000 & 0.984 \\
$C_{3}-C_{6}$ & 0.016 & 0.000 & 0.984
\end{tabular}

[3] K. Atanassov,V. Atanassova, G. Gluhchev, InterCriteria Analysis: ideas and problems. Notes on Intuitionistic Fuzzy Sets 21(1), 81-88 (2015)

[4] V. Atanassova, L. Doukovska, D. Karastoyanov, F. Capkovic, InterCriteria Decision Making Approach to EU Member States Competitiveness Analysis: Trend Analysis, In: Angelov, P., et al. (eds.) Intelligent Systems'2014, Advances in Intelligent Systems and Computing, Vol. 322, 2014, 107-115.

[5] W. Diffe, M. E. Hellman, New direction in cryptography. IEEE Transactions of Information Theory. IT-36, 1976, 644-654.

[6] M. Dorigo, T. Stutzler, Ant Colony Optimization, MIT press, 2004

[7] S. Fidanova, K. Atanassov, P. Marinov, Generalized Nets and Ant Colony Optimization, Academic Publishing House, Bulgarian Academy of Sciences, 2011.

[8] S. Martello, P. Toth, A mixtures of dynamic programming and branchand-bound for the subset-sum problem, Management Science 30, 1984, 756-771.

[9] O. Roeva, S. Fidanova, M. Paprzycki, InterCriteria Analysis of ACO and GA hybrid algorithms, Studies in Computational Intelligence 610, 2016 , $107-126$.

[10] O. Roeva, S. Fidanova, P. Vassilev, P. Gepner, InterCriteria Analysis of a Model Parameters Identification using Genetic Algorithm, Proceedings of the Federated Conference on Computer Science and Information Systems, Vol. 5, 2015, 501-506

[11] A. Sinha, A. A. Zoltner, The multiple-choice knapsack problem, Journal of Operational Research 27, 1979, 503-515. 